\title{
Editorial
}

\section{Application of Plasma Technology in Bioscience and Biomedicine}

\author{
Daniela Boehm ${ }^{1,2}$ and Cristina Canal ${ }^{3,4,5,6, * \text { D }}$ \\ 1 Environmental Sustainability and Health Institute, Technological University Dublin, Grangegorman, \\ D07 H6K8 Dublin, Ireland; Daniela.Boehm@tudublin.ie \\ 2 School of Food Science and Environmental Health, Technological University Dublin, Grangegorman, \\ D07 H6K8 Dublin, Ireland \\ 3 Biomaterials, Biomechanics and Tissue Engineering Group, Department of Materials Science and Engineering, \\ Universitat Politècnica de Catalunya (UPC), c/Eduard Maristany 14, 08930 Barcelona, Spain \\ 4 Research Centre for Biomedical Engineering (CREB), Universitat Politècnica de Catalunya (UPC), \\ c/Eduard Maristany 14, 08019 Barcelona, Spain \\ 5 Barcelona Research Center in Multiscale Science and Engineering, Universitat Politècnica de Catalunya (UPC), \\ 08019 Barcelona, Spain \\ 6 Institut de Recerca Sant Joan de Déu, Santa Rosa 39-57, 08950 Esplugues de Llobregat, Spain \\ * Correspondence: cristina.canal@upc.edu
}

Citation: Boehm, D.; Canal, C. Application of Plasma Technology in Bioscience and Biomedicine. Appl. Sci. 2021, 11, 7203. https://doi.org/ 10.3390/app11167203

Received: 26 July 2021

Accepted: 1 August 2021

Published: 4 August 2021

Publisher's Note: MDPI stays neutral with regard to jurisdictional claims in published maps and institutional affiliations.

Copyright: (c) 2021 by the authors. Licensee MDPI, Basel, Switzerland. This article is an open access article distributed under the terms and conditions of the Creative Commons Attribution (CC BY) license (https:/ / creativecommons.org/licenses/by/ $4.0 /)$.

\section{Introduction}

Plasma technology has been an integral part of research in life sciences for decades through its role in the manufacture and modification of material surface characteristics of many common laboratory consumables, and it is still of interest in many fields, including the treatment of biomaterials and implants. In recent years, the use of plasma at room temperature and atmospheric pressure (cold atmospheric plasma) has led to a shift in the field by putting the focus on biomedicine due to its applicability to heat-sensitive materials, including biomaterials, and expanding the traditional applicability of plasmas to cells and tissues.

Consequently, biomedical applications of cold plasmas have experienced a surge in recent years due to both the evolution of plasma sources to be used under atmospheric pressure and in handy devices, and the increasing need of our society to find novel solutions to unresolved health problems.

The versatility of plasmas and the vibrant advances in the field are reflected in the high number of papers collected in this Special Issue and their wide scope of topics and applicability. Cold plasmas, both at low pressure and at atmospheric pressure, are reflected in the contributions, and the latter being employed both directly on materials and cells, as well as being used to produce plasma-treated liquids which find equivalent applications to plasmas in the different areas mentioned.

In the following sections we briefly discuss the most relevant findings gathered in the papers included in this Special Issue in each of the different areas concerned.

\section{Surface Functionalization \& Coating of Biomedical Materials}

Four papers in this issue are devoted to surface functionalization and plasma coatings for biomedical applications; three of them are more focused on implants, and one of them on materials for cell cultures. In all cases the treatment is aimed at controlling the response of cells in contact with the material, and the different kinds of plasmas employed reflect the high versatility of this technique.

Buxadera and co-workers reported that the atmospheric pressure plasma jet polymerization of a liquid precursor, 2-hydroxyethyl methacrylate, on titanium yielded a coating with enhanced fibroblast adhesion and simultaneously decreased Staphylococcus aureus and Escherichia coli adhesion, which could be relevant for dental implants [1]. A different strategy was employed by Sardella et al., who compared plasma enhanced-chemical vapor deposition (PE-CVD) of polyethylene oxide (PEO)-like coatings generated either at low 
pressure and also aerosol-assisted atmospheric pressure PE-CVD. The atmospheric pressure coatings required an intermediate gradient layer to grant proper adhesion. An accurate modulation of the experimental conditions, both at low and atmospheric pressure, allowed control of the film chemical structure and surface properties, to permanently promote or discourage the cellular adhesion on the surfaces of biomaterials [2].

Focusing on surface functionalization of poly(l-lactic acid) (PLLA), different methods were compared to oxygen plasma by Schieber et al., showing that the oxygenated carboxyl and hydroxyl groups favored endothelial cell adhesion and spreading. This approach was applied to producing 3D printed PLLA bioresorbable stents, which may improve endothelialization after implantation [3].

An interesting work by O'Sullivan et al. investigated the application of collagen coatings onto polystyrene microplates for cell culture using a cold atmospheric pressure plasma process. Compared to standard wet chemical collagen thin films, plasma coatings were thinner and appeared equivalent in terms of coverage, porosity, structure, and chemistry. Significantly, the cold plasma method preserved both the primary and secondary structure of the protein and this resulted in high biocompatibility and cell activity that was at least equivalent to the standard wet chemical technique. The significance of these results is discussed in relation to the benefits of a single step plasma coating in comparison to the traditional multi-step aseptic coating technique [4].

\section{Antimicrobial Applications}

The antimicrobial efficacy of cold plasmas and plasma-treated liquids is of interest in a variety of fields, ranging from the food industry [5] to the medical field [6-9].

For the decontamination of surfaces in the food production industry, plasma-treated water showed efficiency on Pseudomonas fluorescens biofilms, related to a decrease of $\mathrm{pH}$ in the plasma-treated water as the basis for the chemically active components of plasmatreated water and its antimicrobial effects. The findings by Handorf et al. provide valuable insights into the mechanisms of inactivation of biofilms by plasma-generated compounds such as plasma-treated water [5].

More focused on medical applications, Schnabel and co-workers demonstrated the fungicidal effects of plasma-treated water and plasma-processed air on Candida albicans. The authors investigated the possible combination of plasma-treated water and plasmaprocessed air to obtain synergistic effects by combining the former for cleaning and the latter for drying. The authors propose these as an innovative alternative to the sanitization processes currently used in production plants [6]. Also, on the topic of fungicidal effects, in the work by Trebulova et al. the effects of a plasma torch on the inactivation of model yeast (Candida glabrata) provided insights into the effects of the reactive species from the plasma gas phase [7].

Tsoukou et al. investigated the biological activity of plasma-treated water on different biological targets, including both microbial and mammalian cells in vitro. Different trends were observed for cytotoxic activities and effects on enzyme function, which were translated through the selective generation of chemical species and indicate very distinct mechanisms of action which may be exploited when tailoring plasma treated liquids to various applications [8].

Focusing on a specific dental application, the possible use of cold plasma jets on cariogenic films, Figueira et al. recorded reduction in viability both in single- and multi-species biofilms formed by Streptococcus mutans, Streptococcus sanguinis, and Streptococcus gordonii formed on hydroxyapatite discs, simulating the natural bioceramic present in teeth. Single species biofilms were found to be more sensitive to the treatment, showing total elimination for certain strains, but low exposure times of plasma affected both single- and multi-species cariogenic biofilms, which indicates that the treatment is a promising source for the development of new protocols for the control of dental caries [9]. 


\section{Medical Applications}

The versatility of atmospheric pressure plasmas is reflected in the five papers related to medical/therapeutic applications in this issue.

Interesting fundamentals were provided by Ranieri et al. by using glutathione as a marker to establish a correlation between plasma parameters and the resultant liquid chemistry from two distinct sources to predefined biological outcomes. Specific glutathione modifications appeared to be associated with biological responses elicited by plasma. The findings implied that short-lived species and other plasma components were required for the induction of cellular responses, as well as for glutathione modifications. Importantly, the results indicate that comparisons of medical plasma sources should not rely on measurements of long-lived chemical species; rather, modifications of biomolecules (such as glutathione) might be better predictors of cellular responses to plasma exposure [10]. Another paper with a fundamental and methodological focus was provided by Rebl et al., who investigated the effects of plasma on fish cell lines and compared to mammalian cells towards potential applications in wound healing for farmed fish, where skin lesions may occur as a result of intensive fish farming. The authors varied cell density, culture medium, and pyruvate concentration in the medium to conclude that it is mandatory to use similar cell densities and an identical medium, or at least a medium with identical antioxidant capacity, when studying plasma effects on different cell lines. In agreement with this, a standardized protocol for research in plasma medicine can be found in [11]. Altogether, fish cells showed a higher sensitivity towards plasma treatment than mammalian cells [12].

The paper by Belov and co-workers examined the effect of cold plasma on the morphofunctional state of human bone marrow stem cells (BMSC), with particular attention to the dual effects of plasma-induced oxidative stress on the state of stem cells (promotion of proliferative processes vs. destructive processes leading to cell death). The paper discusses the role of mitochondria and reactive oxygen species (ROS) in the cellular response to plasma [13].

With a specific interest in cold plasma jet as a topical skin treatment, Jurov et al. observed that direct plasma treatment was very destructive to mouse skin cells in vitro, leading to low cell viability [14]. This research touches on important points that have been under discussion in the field in recent years and that should be taken into consideration for all further research in this field, the urgent need to: upgrade and standardize existing plasma treatment protocols of cell lines [11]; monitor gas and liquid chemistries; and standardize plasma discharge parameters [14].

With a more specific focus on cancer, Bergemann et al. based their research on the finding that in different tumors, high amounts of hyaluronan (HA) are correlated with tumor progression. Therefore, new tumor therapy strategies are targeting HA production and degradation. The authors found that plasma-treated cell culture medium impaired the HA pericellular coat of $\mathrm{HaCaT}$ cells. This diminished the time-dependent adhesion of cells, although this disturbed HA coat alone was not the reason for the inhibition of cell adhesion [15].

\section{Agriculture}

The applications of plasmas in agriculture is certainly an emerging topic which can have different ramifications, for instance in germination. Ivankov et al. applied different stressors, including cold plasma, to investigate the in vitro germination of industrial hemp cv. Futura 75. Plasma and electromagnetic field treatments improved in vitro seed germination, but had no impact on germination in the field. Cold plasma decreased the content of cannabidiolic acid in hemp leaves by $41 \%$. Vacuum treatment increased the amount of CBDA in female plant inflorescences by $26 \%$ and could be used to increase male plant production, while vacuum treatment stimulated CBD production [16].

As reflected by the different works in this issue, the field of plasma in bioscience and biomedicine has great future prospects for a wide variety of applications, and we will certainly see more exciting advances in the coming years. 
Author Contributions: All authors contribute equally in this paper. All authors have read and agreed to the published version of the manuscript.

Funding: This work was supported by a Science Foundation Ireland Starting Investigator Research Grant (15/SIRG/3466) (DB). This project has received funding from the European Research Council (ERC) under the European Union's Horizon 2020 research and innovation programme (grant agreement No. 714793). CC acknowledges the support of MINECO/FEDER, EU through PID2019103892RB-I00 project and of Generalitat de Catalunya for the ICREA Academia Award and for SGR2017-1165.

Institutional Review Board Statement: Not applicable.

Informed Consent Statement: Not applicable.

Data Availability Statement: Not applicable.

Conflicts of Interest: The authors declare no conflict of interest.

\section{References}

1. Buxadera-Palomero, J.; Fricke, K.; Reuter, S.; Gil, F.; Rodriguez, D.; Canal, C. One-Step Liquid Phase Polymerization of HEMA by Atmospheric-Pressure Plasma Discharges for Ti Dental Implants. Appl. Sci. 2021, 11, 662. [CrossRef]

2. Sardella, E.; Gristina, R.; Fanelli, F.; Veronico, V.; Da Ponte, G.; Kroth, J.; Fracassi, F.; Favia, P. How to Confer a Permanent Bio-Repelling and Bio-Adhesive Character to Biomedical Materials through Cold Plasmas. Appl. Sci. 2020, 10, 9101. [CrossRef]

3. Schieber, R.; Raymond, Y.; Caparrós, C.; Bou, J.; Herrero Acero, E.; Guebitz, G.; Canal, C.; Pegueroles, M. Functionalization Strategies and Fabrication of Solvent-Cast PLLA for Bioresorbable Stents. Appl. Sci. 2021, 11, 1478. [CrossRef]

4. O'Sullivan, D.; McArdle, H.; Ng, S.; Bourke, P.; Forster, R.; O’Neill, L. Deposition of Cell Culture Coatings Using a Cold Plasma Deposition Method. Appl. Sci. 2020, 10, 6670. [CrossRef]

5. Handorf, O.; Pauker, V.; Schnabel, U.; Weihe, T.; Freund, E.; Bekeschus, S.; Riedel, K.; Ehlbeck, J. Characterization of Antimicrobial Effects of Plasma-Treated Water (PTW) Produced by Microwave-Induced Plasma (MidiPLexc) on Pseudomonas fluorescens Biofilms. Appl. Sci. 2020, 10, 3118. [CrossRef]

6. Schnabel, U.; Yarova, K.; Zessin, B.; Stachowiak, J.; Ehlbeck, J. The Combination of Plasma-Processed Air (PPA) and PlasmaTreated Water (PTW) Causes Synergistic Inactivation of Candida albicans SC5314. Appl. Sci. 2020, 10, 3303. [CrossRef]

7. Trebulová, K.; Krčma, F.; Kozáková, Z.; Matoušková, P. Impact of Microwave Plasma Torch on the Yeast Candida glabrata. Appl. Sci. 2020, 10, 5538. [CrossRef]

8. Tsoukou, E.; Delit, M.; Treint, L.; Bourke, P.; Boehm, D. Distinct Chemistries Define the Diverse Biological Effects of Plasma Activated Water Generated with Spark and Glow Plasma Discharges. Appl. Sci. 2021, 11, 1178. [CrossRef]

9. Figueira, L.; Panariello, B.; Koga-Ito, C.; Duarte, S. Low-Temperature Plasma as an Approach for Inhibiting a Multi-Species Cariogenic Biofilm. Appl. Sci. 2021, 11, 570. [CrossRef]

10. Ranieri, P.; Mohamed, H.; Myers, B.; Dobossy, L.; Beyries, K.; Trosan, D.; Krebs, F.; Miller, V.; Stapelmann, K. GSH Modification as a Marker for Plasma Source and Biological Response Comparison to Plasma Treatment. Appl. Sci. 2020, 10, 2025. [CrossRef]

11. Tornin, J.; Labay, C.; Tampieri, F.; Ginebra, M.; Canal, C. Evaluation of the effects of cold atmospheric plasma and plasma-treated liquids in cancer cell cultures. Nat. Protoc. 2021, 16, 2826. [CrossRef] [PubMed]

12. Rebl, H.; Bergemann, C.; Rakers, S.; Nebe, B.; Rebl, A. Plasma Treatment of Fish Cells: The Importance of Defining Cell Culture Conditions in Comparative Studies. Appl. Sci. 2021, 11, 2534. [CrossRef]

13. Belov, S.; Lobachevsky, Y.; Danilejko, Y.; Egorov, A.; Simakin, A.; Maleki, A.; Temnov, A.; Dubinin, M.; Gudkov, S. The Role of Mitochondria in the Dual Effect of Low-Temperature Plasma on Human Bone Marrow Stem Cells: From Apoptosis to Activation of Cell Proliferation. Appl. Sci. 2020, 10, 8971. [CrossRef]

14. Jurov, A.; Kos, ک̌.; Hojnik, N.; Sremački, I.; Nikiforov, A.; Leys, C.; Serša, G.; Cvelbar, U. Analysing Mouse Skin Cell Behaviour under a Non-Thermal kHz Plasma Jet. Appl. Sci. 2021, 11, 1266. [CrossRef]

15. Bergemann, C.; Waldner, A.; Emmert, S.; Nebe, J. The Hyaluronan Pericellular Coat and Cold Atmospheric Plasma Treatment of Cells. Appl. Sci. 2020, 10, 5024. [CrossRef]

16. Ivankov, A.; Nauciene, Z.; Zukiene, R.; Degutyte-Fomins, L.; Malakauskiene, A.; Kraujalis, P.; Venskutonis, P.; Filatova, I.; Lyushkevich, V.; Mildaziene, V. Changes in Growth and Production of Non-Psychotropic Cannabinoids Induced by Pre-Sowing Treatment of Hemp Seeds with Cold Plasma, Vacuum and Electromagnetic Field. Appl. Sci. 2020, 10, 8519. [CrossRef] 